July 21,2006

To appear in Canadian Journal of Philosophy

\title{
Is Classical Electrodynamics an Inconsistent Theory? ${ }^{\dagger}$
}

\author{
Gordon Belot \\ Department of Philosophy \\ University of Pittsburgh
}

\section{Introduction}

Classical Electrodynamics is a classical theory treating systems comprising an electromagnetic field and electrically charged matter. In a recent book Mathias Frisch argues that this theory is inconsistent within in its intended domain of application and that a number of interesting methodological morals follow from this conclusion (2005, pp. 33-35; all citations of Frisch refer to this work).

More precisely, Frisch argues that one can derive a contradiction from the following five premises: (1) there exists a charged particle and an electromagnetic field; (2) the field obeys Maxwell's equation (with the particle providing source terms); (3) the electromagnetic force on the particle is given by the Lorentz force law (for the given electromagnetic field); (4) the particle accelerates; (5) energy is conserved. He goes on to argue that the inconsistency of (1)-(5) has the following fairly direct consequences: (a) there exist inconsistent theories; (b) consistency is a theoretical virtue like any other; (c) we have here a victory for the models-based approach to philosophy of science far away from its home territory of application and approximation; and (d) we must reform our notion of theory acceptance.

\footnotetext{
${ }^{\dagger}$ A previous version of this paper was presented at the $42^{\text {nd }}$ Annual Conference of the Western Canadian Philosophy Association. Many thanks to Laura Ruetsche and Steve Weinstein for helpful comments and, especially, to Mathias Frisch for several very helpful conversations.
} 
My purpose here is to discuss Frisch's argument for the inconsistency of electromagnetism and the conclusions he draws from it. I contend that although the argument is valid it relies on a contentious understanding of the Lorentz force law. So I think that there is plenty of room to be sceptical of Frisch's conclusion. I also contend that even if this conclusion is granted, the connection with the methodological consequences (a)-(d) is not nearly so tight as Frisch takes it to be.

Sections II and III address preliminary matters concerning the framework of classical electrodynamics. One of the topics addressed is the nature of particles in classical electrodynamics. Another is an important ambiguity in the construal of the Lorentz force law: one has to decide whether to employ the total field or only the field external to the particle in applying this law. This ambiguity is crucial: under one reading of the law, (1)-(5) above are consistent; under the other they are inconsistent (Frisch favours this latter reading). Sections IV and V are devoted to these points. Section VI weighs the question which version of the law we should take to be constitutive of classical electrodynamics. Here I differ from Frisch, and so I conclude that classical electrodynamics is consistent. In Section VII I turn to the question whether there is in fact a tight connection between Frisch's methodological morals and his thesis that classical electrodynamics is inconsistent.

Despite the critical tone of this paper, I want to emphasise that there is much that I admire in Frisch's book - and to encourage people to read it to see how fruitful for philosophy of physics and for philosophy of science engagement with less than fundamental theories can be. My aim here is only to deflate Frisch's discussion of inconsistency. 


\section{The Maxwell-Lorentz Equations}

The fundamental equations of the classical electrodynamics are Maxwell's equation and the Lorentz force law, with the latter coming in two versions.

MaXWell's Equations. Choosing an inertial frame, we can decompose the electromagnetic field into the electric field $\mathbf{E}$ and the magnetic field $\mathbf{B}$. Then we can write Maxwell's equations as:

$$
\begin{aligned}
& \nabla \cdot \mathbf{E}=\rho \\
& c \nabla \times \mathbf{E}=-\partial_{t} \mathbf{B} \\
& \nabla \cdot \mathbf{B}=0 \\
& c \nabla \times \mathbf{B}=\mathbf{J}+\partial_{t} \mathbf{E} .
\end{aligned}
$$

Here $\rho$ and $\mathbf{J}$ are the charge and current density corresponding to some given configuration of charged matter in spacetime, and c is the velocity of light.

THE LORENTZ FORCE LAW FOR THE TOTAL FIELD. This version of the Lorentz force law reads

$$
\mathbf{F}_{\mathrm{EM}}=q(\mathbf{E}(\mathbf{x})+\mathbf{v} \times \mathbf{B}(\mathbf{x}))
$$

It determines the electromagnetic force that a particle of charge $q$ and velocity $\mathbf{v}$ located at spacetime point $\mathbf{x}$ feels in the presence of electric field $\mathbf{E}$ and magnetic field $\mathbf{B}$.

THE LOREnTZ ForCe LAW FOR THE EXTERnAL FIELD. This version of the Lorentz force law reads

$$
\mathbf{F}_{\mathrm{EM}}=q\left(\mathbf{E}_{\mathrm{Ext}}(\mathbf{x})+\mathbf{v} \times \mathbf{B}_{\mathrm{Ext}}(\mathbf{x})\right)
$$


It determines the electromagnetic force that a particle of charge $q$ and velocity $\mathbf{v}$ located at spacetime point $\mathbf{x}$ feels in the presence of external electric and magnetic fields $\mathbf{E}_{\mathrm{Ext}}$ and

$\mathbf{B}_{\mathrm{Ext}}$ (i.e., $\mathbf{E}_{\mathrm{Ext}}$ is $\mathbf{E}$ minus the field generated by the particle located at $\mathbf{x}$, and similarly for $\left.\mathbf{B}_{\mathrm{Ext}}\right)$.

So the equations governing the behaviour of the field require as input a fixed distribution of charged matter; while the equations governing the motion of charged matter (in either the total-field form or the external-field form) require as input a fixed configuration of the electromagnetic field. The difference between the two versions of the Lorentz force law is this: in the first version, but not in the second, in calculating the electromagnetic force on a particle one takes into account the electromagnetic field generated by the particle itself.

Our question whether classical electrodynamics is consistent can be recast as the question whether there are any joint solutions to Maxwell's equations and the Lorentz force law in which a particle accelerates and energy is conserved. I will be more precise below about what this means. But we can see already that there will be two construals of the question of the consistency of classical electrodynamics, corresponding to the two versions of the Lorentz force law.

\section{Particles in Classical Electrodynamics}

For present purposes we may restrict attention to situations in which the electromagnetic field interacts with a single particle of charge $q \neq 0$. But what is a particle in the context of classical electrodynamics? 
The simplest option is to take our particle to be a point particle. This leads to various well-known difficulties. On the one hand, there is no well-defined energy for a system consisting of an electromagnetic field and a point particle (see, e.g., Feynman et al. 1964, §8-6). On the other, the external-field version of the Lorentz force law breaks down in the context of point particles (see Section IV below).

We encounter problems of a different sort if we consider extended particles. Here the simplest option is to take our particle to be rigid in some preferred frame and to take the charge to be smeared throughout the particle or over its surface. There must be powerful forces at work to maintain the shape of such a particle-since the infinitesimal charges composing it will naturally tend to repel one another. Absent an account of such a particle-and an account of how the dynamics of such an object determines the preferred frame in which it is rigid-rigid extended charged particles are hardly all one could wish for. ${ }^{1}$

So there is no notion of particle ideally suited to classical electrodynamics. Of course, this is a fact that physicists had to face up to long ago-the early twentieth century quest for a classical theory of the electron foundered on the considerations mentioned above.

I think the most sensible attitude is that the Maxwell-Lorentz equations no more pick out a structure for microscopic charged particles than Newton's law of gravity picks out a structure for microscopic massive particles: one is free to stipulate a notion of particle for a given investigation - and one nor more needs to provide an account of what

\footnotetext{
${ }^{1}$ Similar points can be made about other types of extended charged particles. Here an important case is the relativistically rigid particle, which maintains its shape in its instantaneous rest frame. For a modern approach to such particles and references to the literature, see Spohn (2004, especially §2.5). See Frisch (p. 57) for worries about such particles.
} 
holds such a particle together for the purposes of studies within classical electrodynamics than Euler needed to provide an account of what held rigid bodies together for the purposes of his studies of within classical mechanics. So I am happy with treatments involving point particles - so long as the equations of motion make sense and the notion of energy is not invoked. And I am equally happy with treatments involving rigid extended particles. I am not concerned that rigid particles are nonrelativistic, since my present concern is with the question whether (1)-(5) are consistent-and special relativistic considerations form no part of (1)-(5).

In setting up his argument for the inconsistency of classical electrodynamics in Chapter 2 of his book, Frisch appears to adopt a similarly liberal view: he is careful to demand only that there exist "discrete, finitely charged accelerating particles" (p. 33) - leaving it open whether these particles are point particles or extended particles of small size. $^{2}$

REMARK 1. In Chapter 3 Frisch sometimes sounds as if he thinks that particles in classical electrodynamics are necessarily point particles. ${ }^{3}$ But this view is in serious tension with his treating the principle of the conservation of energy as constitutive of classical electrodynamics (i.e., in using this principle as a premise in his argument for the inconsistency of the theory). For, I would assume, even if classical electrodynamics is an inconsistent theory, the notion of the energy of a field-particle system must at least sometimes be defined if conservation of energy is constitutive of the theory. So I will

\footnotetext{
${ }^{2}$ For passages in which it seems that for Frisch a discrete particle may be extended, see pp. 51, 52, and 69.

${ }^{3}$ See pp. 47 and $66 \mathrm{f}$.
} 
assume that under Frisch's view classical electrodynamics includes models with extended charged particles.

\section{The Consistency of Classical Electrodynamics (Total-Field Version)}

Let us now turn to the question of the consistency of classical electrodynamics and begin by considering the case where the Lorentz force felt by a particle is determined by the total field. And let us suppose that given a particle history, we can construct the distribution of charge and current associated with that history and determine the electromagnetic force experienced by the particle at each instant. ${ }^{4}$ We say that an electromagnetic field configuration on spacetime (for short: a field) is a solution to Maxwell's equations for a given particle history if the field solves Maxwell's equations for the charge and current density corresponding to that particle history. And we say that a given particle history is determined by the Lorentz force law for a given total field if the electromagnetic force experienced by the particle at each instant is given by the Lorentz force law for the given total field. Let us call a pair (field, particle history) a solution to the joint Maxwell-Lorentz equations in the first sense (for short, a solution to $M-L_{1}$ ) if the given field is a solution to Maxwell's equations for the given particle history while the given particle history is determined by the Lorentz force law for the given total field.

We are interested in the question whether $M-L_{1}$ has any solutions in which the particle accelerates and energy is conserved.

It is simplest and most natural to take our charged particle to be a point particle. Unfortunately, the total-field version of the Lorentz force law ceases to make sense in the

\footnotetext{
${ }^{4}$ To accomplish the latter task for rigid or point particles we proceed as follows: from the particle history, we can determine the acceleration at each instant; this allows us to determine the total force acting on the particle; the electromagnetic force can be found by subtracting off all non-electromagnetic forces.
} 
context of point particles. A history of a point particle is given by specifying the mass and charge of the particle along with its worldline in spacetime. Now, if we ignore other electromagnetic radiation and take our particle to be at rest, then the first Maxwell equation, $\nabla \cdot \mathbf{E}=4 \pi \rho$, is essentially just Coulomb's law, telling us what kind of forces a charged test particle would feel due to the charge of our given particle (see Jackson 1962, pp. 1-7). Like the force of gravitation, this force goes inversely as the square of the distance between the particles - and so it blows up as we approach our given particle. But in order to determine how our particle moves we need to know the values of $\mathbf{E}$ and $\mathbf{B}$ at the spacetime point at which our particle is located. So it looks like we cannot make any sense of the total-field version of the Lorentz force law in the context of point particles-positing such particles undermines the rules of the game.

This a little bit too hasty but not too far wrong. A more careful way of putting the point of the preceding paragraph would be to say that if one employs point particles, then one has to treat the charge density and current not as ordinary functions on spacetime but as distributions; and one has to expect that the electric and magnetic fields will likewise make sense only as distributions, since they will have singularities along the particle's worldline. ${ }^{5}$ Now, moving from functions to distributions need not spell disaster. Indeed, given distributional source terms, one can make sense of Maxwell's equations and look for distributional solutions. But in general, if one is using distributions one can make sense only of equations in which one does not take products of distributions. Maxwell's equations satisfy this condition but the joint Maxwell-Lorentz equations do not because

\footnotetext{
5 The prototypical distribution that is not a function is the Dirac $\delta$-function, which can be thought of as vanishing everywhere except at the origin, but which nonetheless has a nonvanishing integral. In our case: the distributions representing the charge and current density would vanish everywhere except on the particle worldline, but would nonetheless have a nonvanishing integral over any region overlapping with the particle worldline.
} 
the Lorentz force law involves the product of the particle velocity with the magnetic field. Under present assumptions, these quantities are both distributional - so for the time being, one does not know how to make good mathematical sense of $M-L_{1}$ for a point particle. $^{6}$

So let us consider $\mathrm{M}-\mathrm{L}_{1}$ applied to rigid extended charged particles. We assume that the particle maintains a spherical shape in a given inertial frame. In order to give sense to the question whether a pair (field, particle history) is a solution to the joint Maxwell-Lorentz equations when the particle involved is an extended particle, we have to spell out how the history of such a particle is represented, how such a history determines the charge and current densities, and how one applies the Lorentz force law to such a particle.

Our spherical particle is charged. We assume that the charge density associated with the particle is constant in time, is spherically symmetric within the particle (and vanishing outside), is smooth, and integrates to $q$. So we can represent a history of the particle by giving the worldline of its centre of mass. And this determines the charge density $\rho$ on spacetime associated with the particle history, which in turn determines the current density $\mathbf{J}$ associated with the particle history (here the current density at an instant is just given by the product of the charge density at that instant with the velocity $\mathbf{v}$ of the particle at that instant).

How are we to apply the Lorentz force law to our extended particle? Rigid bodies are funny things - clearly there must be forces that hold them together, but one is allowed to ignore these because they do no work. But if we have a rigid body undergoing external

\footnotetext{
${ }^{6}$ Extant schemes for handling products of generalized functions all appear to involve some undesirable arbitrariness. See Hörmann and Kunzinger (2000) for a treatment of a two-dimensional system modelled on the Maxwell-Lorentz system with point particle source terms.
} 
forces, with these forces perhaps being stronger on some parts of the body than on others, what are we to do? In this case, we calculate the external force acting on each part of the body; adding up all such forces (or integrating when as in this case the body has continuum many parts) gives us the total external force acting on the body which we then apply to the centre of mass to calculate the motion of the body as a whole (see Whittaker 1947, p. 127 f.). So we calculate the Lorentz force corresponding to a given field by calculating the infinitesimal Lorentz force at each point of the particle, and integrating these forces over the points of the body to give a total Lorentz force that is applied to the centre of mass of the body.

A pair (field, particle history), with the particle involved one of our rigid charged spheres, is a solution of M-L $\mathrm{L}_{1}$ if: (i) the field solves Maxwell's equations for the charge and current density determined by the particle history; (ii) the Lorentz force law for the given total field (applied as described above) gives the electromagnetic force acting on the particle at each instant.

There has been renewed interest in $\mathrm{M}-\mathrm{L}_{1}$ for an extended rigid particle among mathematicians in recent years. It has recently been proven that solutions exist and are unique for a wide range of initial data and that the total energy of the field-particle system is conserved in time. ${ }^{7}$ The set of solutions includes situations in which the particle accelerates. $^{8}$

\footnotetext{
${ }^{7}$ See Bauer and Dürr (2001, Theorem 1 and Lemma 5) or Komech and Spohn (2000, Proposition 2.3). Here the total energy of the field-particle system is the sum of the kinetic energy of the particle with the usual energy of the field (i.e., the integral over space of $\mathbf{E}^{2}+\mathbf{B}^{2}$ ).

${ }^{8}$ In the case where no external forces act, Komech and Spohn allow any initial data consistent with the Maxwell equations, so long as the components of the fields are square-integrable; the restrictions of Bauer and Dürr are similarly weak.
} 
So conditions (1)-(5) are consistent when one employs the total electromagnetic field in calculating the Lorentz force felt by a particle.

REMARK 2. For an overview of recent literature on $\mathrm{M}-\mathrm{L}_{1}$ with rigid charged particles, see Spohn (2004). Note that the framework can be extended to cover multiple particles and particles which rotate. The Coulomb force law and Dirac equation for the motion of a charge in a fixed field emerge as appropriate limits. Note that in order to treat various approximations, authors in this literature sometimes introduce a fixed time-independent electromagnetic field that does not react to the charged particle but which does influence it via the Lorentz force law. It must be emphasized that this is for the purpose of approximation only: the Maxwell-Lorentz equations (under the present understanding) describe a genuine interaction between the electromagnetic field and a charged particle that already treats the self-field of the particle. Other than for the purpose of constructing approximations there is in no need in this framework to treat the external field and the self-field of the particle separately.

\section{The Inconsistency of Classical Electromagnetism (External-field Version)}

Let us now turn to the version of the Lorentz force in which only the field external to a particle is used in calculating the Lorentz force felt by that particle.

Let us suppose that given a particle history: (i) we can construct the charge and current densities determined by the particle; (ii) we can determine the electromagnetic force acting on the particle at each instant; and (iii) for any total electromagnetic field we 
can effect a decomposition into the external field and the self-field of the particle. ${ }^{9}$ Then a pair (field, particle history) is a solution of $M-L_{2}$ if: (a) the field solves Maxwell's equations for the charge and current density corresponding to the particle; and (b) the electromagnetic force on the particle at each instant is given by the Lorentz force law, taking into account only the external part of the field.

Solutions of $\mathrm{M}-\mathrm{L}_{2}$ can be thought of as arising in the following way: first one specifies the external field; this is plugged into the Lorentz force law to give the motion of the particle; the self-field of the particle is the purely retarded solution to the Maxwell equations for the charge and current density corresponding to the particle; the total field is the sum of the external field and the self-field. Thus physicists calculate the radiation given off by a particle in a synchrotron by first finding that a charged particle moving in the powerful magnetic field inside such a device follows a circular orbit, then identifying the radiation of the particle with the external field of a particle with this motion.

As Frisch points out (pp. 33-35) energy is not conserved in solutions to $M-L_{2}$ in which the particle undergoes acceleration. This is easiest to see in the case of the synchrotron: since the particle is giving off radiation, its kinetic energy ought to be decreasing; but because we use only the external field in calculating the Lorentz force, the particle moves in a permanent circular orbit, with constant kinetic energy. The argument can be extended to cover cases in which the particle's kinetic energy is changing. ${ }^{10}$

\footnotetext{
${ }^{9}$ Schematically, we accomplish (iii) as follows: the self-field is the purely retarded solution to Maxwell's equations for the charge and current density determined by the particle; the external field is the difference between the self-field and the total field.

${ }^{10}$ Suppose that the Lorentz force is the only force acting on the particle during some period of acceleration. Then, on the one hand, by the definition of work, the change in kinetic energy during this period is just equal to the work done on the particle by the field. On the other hand, by conservation of energy, since the
} 
So (1)-(5) of Section I above are indeed inconsistent if we adopt the external-field form of the Lorentz force law.

\section{Consistent or Inconsistent?}

The question whether classical electrodynamics is inconsistent turns upon which version of the Lorentz force law we adopt. If one adopts the first version of the Lorentz force law, which employs the total field, then conditions (1)-(5) of Section I above are consistent; if one adopts the second version, which employs the external field, then they are inconsistent. Frisch takes the second version as constitutive of classical electrodynamics. Should we follow him in this?

The case in favour of the total-field version of the law is straightforward. The framework that results if we adopt the external-field version of the law looks like an approximation: we take only part of the total field into account in solving for the particle's motion. Prima facie, one would think that the total-field version of the law should be preferred, as taking into account all of the actors involved. And the fact that energy conservation is violated in situations in which an accelerated particle is governed by the external-field version of the law, but not when the total field is taken into account, seems to provide reinforcement for the intuition that the total-field version of the law is fundamental while the external-field version is something that is naturally seen as arising in the course of making a useful approximation. A further consideration is that the $M-\mathrm{L}_{1}$ arises from a very natural Lagrangian - and so admits of quantization - while $M-\mathrm{L}_{2}$

particle is involved in two energetic processes with two distinct partners (acceleration by the Lorentz force determined by the external field and radiation to the self-field) the change in kinetic energy should be the work done minus the energy radiated; since the latter is positive for an accelerating charged particle, we have a contradiction. 
presumably does not admit a natural Lagrangian treatment (since energy is not conserved in this theory, any Lagrangian for it would have to be time-dependent and so break the symmetries of the background spacetime). ${ }^{11}$

What is there to be said in favour of taking the external-field version of the Lorentz force law as constitutive of classical electrodynamics? The big advantage of this version is that it allows one to work with much simpler equations. One begins with a given field and solves for the motion of the particle by treating it as a test particle (acted on by the field but not acting upon it). One then takes this motion as given and solves for the field generated by the particle by treating it as a test field (acted upon by the particle but not acted upon by it). In this way one avoids having to solve the nonlinear joint Maxwell-Lorentz equations. One benefit is immediate: one can treat point particles within this framework (each of the equations one must solve in this approach is wellformed if one takes the external field to be smooth, even if the source terms are distributional).

As I understand them, Frisch's reasons for viewing the external-field version of the Lorentz force law as constitutive of classical electrodynamics are rooted in a desire to be faithful to the practice of physicists, as embodied in the textbook literature. For instance, in Jackson's canonical textbook one finds the Lorentz force law given in the external-field form (1962, p. 191). And one finds there also many problems in which the motion of a charged body in an electromagnetic field is handled via the external-field version of the Lorentz force law. One even finds analyses of phenomena such as synchrotron radiation and Bremsstrahlung that proceed in the characteristic stepwise

11 Thanks to Steve Weinstein for this point. For the Lagrangian treatment of $M-L_{1}$ and for the corresponding quantum theory, see Spohn (2004). 
fashion underwritten by $\mathrm{M}-\mathrm{L}_{2}$ : first an external field is prescribed; then one solves for the motion of a particle in this field; then one solves for the total field by calculating the radiation emitted by these charges. So it appears that at the level of official doctrine and at the level of problem-solving, the external-field version of the Lorentz force law is taken as standard by physicists.

I don't myself find this consideration decisive. As all philosophers who have turned to them know, physics textbooks do not have as a pre-eminent goal logical care in the exposition of their subject matter. It often isn't difficult to find what one might politely describe as serious tensions in the expositions of even the best textbooks - a fact that does not at all detract from their pedagogical quality. This leaves philosophers who want to base judgements of the content of a given physical theory upon the textbook literature in a dilemma: one can either engage in rational reconstruction, or one can conclude the content of the theory is indeterminate.

So it is in texts on classical electrodynamics. Jackson begins his final chapter as follows:

In the preceding chapters the problems of electrodynamics have been divided into two classes, one in which the sources of charge and current are specified and the resulting electromagnetic fields are calculated, and the other in which the external electromagnetic fields are specified and the motions of charged particles or currents are calculated. ... Occasionally, as in the discussion of bremsstrahlung, the two problems are combined. But the treatment is a stepwise one-first the motion of the charged particle in an external field is determined, neglecting the emission of radiation; then the radiation is calculated from the trajectory as a given source distribution.

It is evident that this manner of handling problems in electrodynamics can be of only approximate validity. ... A correct treatment must include the reaction of the radiation on the motion of the sources. (pp. $578 \mathrm{f}$.)

Earlier in the book Jackson appears to identify classical electrodynamics with $\mathrm{M}-\mathrm{L}_{2}$. If this is correct, then Jackson ought to view the treatments he has offered of synchrotron radiation and Bremsstrahlung as featuring exact solutions to fundamental equations of classical electrodynamics. But here he says that these treatments are of only approximate 
validity (and this is supposed to be obvious - not a surprising revelation of quantum theories). It would seem that he must mean either that they make only approximately correct predictions - or that $\mathrm{M}-\mathrm{L}_{2}$ represents a mere approximation scheme within to the full theory of classical electrodynamics. Since Jackson goes on following the passage quoted to argue that one expects the analyses he has give to be empirically adequate down to the level at which quantum effects begin to appear, it seems to me that we must read him as here disowning the external-field reading of the Lorentz force law.

One can find a passage playing a similar role in another classic textbook, Feynman et al. (1964). Feynman does not explicitly adopt the external-field version of the Lorentz law, as Jackson does. But like Jackson Feynman spends much of his time solving problems in which one ignores the self-field of a moving charged body. Much that he says gives the impression that he identifies classical electrodynamics with $\mathrm{M}-\mathrm{L}_{2}{ }^{12}$ But he too eventually turns to the modifications of electrodynamics that have been suggested in light of the well-known difficulties of the theory. ${ }^{13}$ One of the proposals he consider involves taking the external-field version of the Lorentz force laws as the law governing the motion of charged matter in an electromagnetic field. This already strongly suggests that he has not meant to be all along identifying classical electrodynamics with $\mathrm{M}-\mathrm{L}_{2}$. This suggestion is reinforced by what he goes on to say:

We must say immediately that such theories require a modification of the idea of the electromagnetic field. You remember we said at the start that the force on a particle at any point was determined by just two quantities - E and $\mathbf{B}$. If we abandon the "self-force" this can no longer be true, because if there is an electron in a certain place, the force isn't given by the total $\mathbf{E}$ and $\mathbf{B}$, but by only those parts due to other charges. So we have to keep track always of how much of $\mathbf{E}$ and $\mathbf{B}$ is due to the charge on which you are calculating the force and how much is due to the other charges. (p. 28-6)

\footnotetext{
12 Thanks to Mathias Frisch for emphasizing this to me.

13 Feynman discusses a number of such difficulties. Each of them can be traced to infinities resulting from the postulation of point particles or to the clash between Lorentz invariance and the postulation of rigid electrons. See Feynman et al. (1964, pp. 28-1 ff.).
} 
Feynman certainly appears to think that the total-field version of the Lorentz force law is the canonical one and that the external-field formulation is an unorthodox one-if taken as exact rather than as part of an approximation procedure.

I think we should take these remarks of Jackson and Feynman at face value: their considered view is that within classical electrodynamics the total field, not just the external field, must be taken into account in calculating the force experienced by a charged body moving in an electromagnetic field. This leaves me with something to explain away: the nonchalant manner in which these authors employ the external-field version of the law in solving problems. I explain it away thus: the full Maxwell-Lorentz equations $\mathrm{M}-\mathrm{L}_{1}$ are non-linear and very difficult to work with; since one expects to get very accurate predictions by using the merely approximate $M-L_{2}$, textbook authors adopt the latter in the service of pedagogy; this is harmless so long as they are careful-as Jackson and Feynman eventually are - to warn readers that something is being swept under the rug.

Everyone will grant that there are serious tensions in Jackson's and Feynman's discussions. I think that we should take most seriously their explicit remarks concerning the shortcomings of the external-field version of the Lorentz force law and explain away their reliance on it in solving problems. Frisch thinks we should do the opposite. This is an interesting suggestion. But I, for one, worry that this advice will lead us to some strange conclusions because I think that physicists are very often (and very reasonably) willing to be sloppy about what is approximate and what is exact in explaining material to students - and this sloppiness will make it very easy for someone who proceeds as Frisch advises to conclude that any reasonably complex physical theory is inconsistent. 
Consider any theory $\mathrm{T}$ treating two kinds of system interact in a mathematically intractable way: (i) physicists will illustrate the content of the theory by asking students to consider cases in which the first type of system is treated as fixed while the second is treated dynamically, and vice versa; (ii) let $\mathrm{X}$ be some desirable property of $\mathrm{T}$ that is not possessed by all of the solutions arising at stage (i); (iii) taking $\mathrm{X}$ to be constitutive of $\mathrm{T}$ as well as the two approximation schemes appearing in (i) will lead to the conclusion that $\mathrm{T}$ is inconsistent. ${ }^{14}$ In this way you can show that just about any theory is inconsistent, if you insist on fidelity to the problem-solving practice of physics. This conclusion should be unwelcome to everyone. ${ }^{15}$ So I am very suspicious of Frisch's suggested procedure for isolating the content of theories - and I am not tempted to apply it to the case of classical electrodynamics.

I do not see in the practice of physicists the same clear-cut evidence that Frisch sees that classical electrodynamics is founded upon the external-field version of the Lorentz force law rather than the total-field version. Indeed, I think that many physicists would agree with me that that total-field version is fundamental while the external-field version is part of an approximation procedure. And so I cannot follow Frisch in concluding that classical electrodynamics is an inconsistent theory.

REMARK 3. In $\$ 3.1$ of Chapter 3 of his book, Frisch considers the view that classical electrodynamics as he conceives of it should be viewed as a mere approximation to a

\footnotetext{
${ }^{14}$ Of course $I$ think that this is just how Frisch reaches his conclusion: he begins with classical electrodynamics; he fixes attention on the approximation in which it is safe to treat the field as given and on the approximation in which it is safe to think of the charge and current as given; he then notes that treatments arising in this way are inconsistent with the conservation of energy - surely itself a component part of classical electrodynamics. This secures the conclusion that the theory is inconsistent.

${ }^{15}$ Certainly it should be unwelcome to Frisch. For there is no is no reason that $\mathrm{T}$ above could not be fundamental. And Frisch holds that inconsistent theories are not possible objects of belief and so are not plausible candidates to be fundamental theories (p. 43).
} 
theory involving rigid extended charged particles. He conceives of this as the question whether physicists should prefer a theory of the latter type to the theory founded upon M$\mathrm{L}_{2}$ and involving only point particles. ${ }^{16} \mathrm{He}$ concludes that they should not since (i) there is no physically sound account of the forces holding rigid charges rigid, (ii) nor is there any account of how such bodies can be reconciled with Lorentz invariance.

I am happy to grant (i) and (ii). But I note that these reasons are reasons to prefer point particles to extended particles rather than to prefer $M-L_{2}$ to $M-L_{1}$. And as noted above (see Remark 1) if Frisch wants to employ the principle of conservation of energy in his argument that classical electrodynamics is inconsistent, then it is illicit to debar extended particles from classical electrodynamics (since the energy of a field-particle system is well-defined only for such particles). So these considerations do not seem to me to tell against the view adopted here that the models discussed in Section IV above show that classical electrodynamics is consistent.

\section{Methodological Morals?}

On Frisch's view the argument for the inconsistency of classical electrodynamics is of interest in part because of its tight connection with the following methodological morals:

(a) there exist inconsistent theories; (b) consistency is a theoretical virtue like any other;

(c) we have here a victory for the models-based approach far away from its home territory of application and approximation; and (d) we must reform our notion of theory acceptance.

\footnotetext{
${ }^{16}$ As noted above (see Remark 1), a slide takes place between Chapter 2 and Chapter 3 of Frisch's book: whereas in Chapter 2 extended particles are allowed in classical electrodynamics, in Chapter 3 they are not.
} 
In this section I question the strength of the connection between the claim about inconsistency and these morals. For this purpose it is convenient to bracket the considerations discussed above and to reason as if the external-field version of the Lorentz force law were the correct one. That is, I proceed as if (1)-(5) were indeed inconsistent.

I consider each of Frisch's conclusions (a)-(d) in turn.

(a) There exist inconsistent theories. Frisch takes this to follow directly from the inconsistency proof. And he thinks it follows that there is something wrong with standard accounts which require theories to be consistent (pp. 5-12). But he is wary that some readers will resist this point-digging in their heels and insisting that classical electrodynamics as embodied in (1)-(5) does not deserve to be called a theory precisely because it is inconsistent.

I am one of those readers. 'Theory' in the hands of philosophers of science is a technical term - an explication of a term in use. I am granting for present purposes that (1)-(5) are inconsistent; and I am happy to grant that they jointly form the foundation of what physicists call 'the theory of classical electrodynamics.' But in choosing a sense for the term 'theory' in philosophy of science, we have to trade off between fidelity to common use and fecundity of the explicans. I think there is a lot to be said for sticking to the traditional use, which allows one to treat a theory as a consistent set of sentences or as a set of solutions to some equations. And this seems to me to outweigh the attractions of being true to the usage of physicists in a case like this. What then do I make of the physicists' practice of treating classical electrodynamics as characterized by Frisch as a 
single theory? I see here two well-behaved classical theories: one features Maxwell's equations and is employed to model field-particle systems in contexts where the evolution of the particles can be taken as given; the other features the Lorentz force law and is employed to model field-particle systems in contexts where the evolution of the field can be taken as given. ${ }^{17}$ Since the inconsistency of (1)-(5) is being taken for granted, I have to deny that there is any classical theory that can handle genuine interactions between the field and particles. But that is okay-situations in which one expects both of my theories to break down are situations in which one expects quantum effects to matter (p. 42).

Now, Frisch is unlikely to approve of my curmudgeonly notion of theory. But he explicitly claims that his methodological morals go through whether or not we think that the conjunction of (1)-(5) deserves to be called a theory (pp. 19, 26 f.). So I propose to evaluate (b)-(d) by seeing what the claims themselves and the arguments for them look like when translated from his preferred terminology to mine.

(b) Consistency is a theoretical virtue like any other. On Frisch's view, most physicists prefer the package (1)-(5) to its consistent competitors (e.g., Dirac's equation for the motion of the classical electron with its funny runaway solutions, etc.). So on this view we sometimes prefer an inconsistent theory that is highly virtuous in other respects (simplicity, accuracy, etc.) to a consistent theory that is less virtuous in these other respects. As noted above, this conclusion applies only to non-fundamental theories.

\footnotetext{
${ }^{17}$ Note that, intuitively, synchrotron radiation provides a case falling under both theories: given the strength of the field inside the device it is safe (but not perfectly accurate) to treat this field as fixed in calculating the motion of the particles; once this motion is found it is safe (but not perfectly accurate) to treat it as given and calculate the radiation given off by the particles. So I endorse the usual analysis as a sensible approximation. As noted above, it is not obvious how an advocate of Frisch's view can rationalise our sense that approximation is involved here.
} 
Translated into my idiolect, the moral is: in non-fundamental contexts we sometimes prefer to treat a given class of phenomena via a cluster of pair-wise inconsistent theories rather than with a single theory - when the former seems superior in respects such as simplicity, accuracy of prediction, etc. I wholly agree: at the classical level a gas is sometimes treated as a box of atoms, sometimes as a fluid; the two sorts of treatment are inconsistent with one another; but we prefer deploying such a team of theories to deploying just one of them, or to any single consistent theory that we know of. So (b) is very plausible when translated into the idiolect of someone who insists that theories are consistent. But it is then revealed as making a familiar point (although it may nonetheless be surprising to have it made about theories covering electromagnetic phenomena).

(c) We have here a victory for the models-based approach far away from its home territory of application and approximation. The central thesis of the models-based approach is that "a theory's laws do not determine the models that scientists use to represent the phenomena" (p. 10). The laws of an inconsistent theory cannot on their own single out a nontrivial set of representations, since there are no solutions of the theory's fundamental equations that represent the phenomena the theory is used to model. On Frisch's view, since we do use classical electrodynamics to represent physical phenomena despite the inconsistency of the theory, something other than its laws is playing a role in determining the representational capacity of the theory.

How is this supposed to work? Frisch introduces (p. 6) a distinction between the model-theoretic models of a theory (the solutions to its equations) and the representational models of a theory (objects, abstract or otherwise, associated with the 
theory that can be used to represent the world). The picture is that an inconsistent theory like classical electrodynamics has representational models even though it has no modeltheoretic models. If this is so, then any inconsistent theory must be more than just a set of laws: it must include further principles that pick out these representational models. In the case of classical electrodynamics, these further principles tell us that the representational models arise as solutions to Maxwell's equations for given particle motions and as particle motions obeying the Lorentz force law for given field configurations. So the models-based approach is required to make sense of the practice of classical electromagnetism, even in the most theory-dominated contexts (p. 12).

Translated into my idiolect the picture is as follows: when we employ a cluster of mutually inconsistent theories to a treat a given class of phenomena, we cannot expect to generate the associated models by looking for solutions to the conjunction of the equations of all of these theories. Rather, there are some principles that tell us to look for solutions to the equations of each of the theories taken on their own, and to view these as having representational capacity. But this is just to say: we can get away with thinking of each of these theories as determining its class of models in the usual way-i.e., as generating the set of solutions to the equations of the theory. We will of course need further principles that demarcate the domain of applicability of each theory-but every non-fundamental theory involves such principles. So the argument given doesn't seem to support the models-based view if I am allowed to use my sense of 'theory' throughout, including in the characterization of the models-based approach. 
I call this one a draw: whether there is any advantage for the models-based account here depends on which sense of 'theory' its proponents have in mind in laying out their view.

(d) We must reform our notion of theory acceptance. Regarding van Fraassen's notion of acceptance, Frisch observes: "If accepting a theory entails being committed to the literal truth of its empirical consequences, then accepting an inconsistent theory entails being committed to inconsistent sets of consequences" (p. 41). Frisch recommends replacing van Fraassen's notion of acceptance with the following: "in accepting a theory, our commitment is only that the theory allows us to construct successful models of the phenomena in its domain, where part of what it is for a model to be successful is that it represents the phenomena at issue to whatever degree of accuracy is appropriate in the case at issue" (p. 42).

Now: in van Fraassen's scheme acceptance of a theory involves belief that the theory saves the phenomena-that it has a model that correctly represents the facts about observable things (1980, p. 12). Since it is clear that van Fraassen has model-theoretic models in mind $(1980,43)$, we need to do something to adapt his notion of acceptance to the case of inconsistent theories which have no model-theoretic models. The solution is obvious: we can require that accepting a theory entail belief that the theory has a representational model that correctly represents the observable facts. But this actually seems a bit too demanding, since inconsistent theories are only accepted in nonfundamental contexts. In such contexts, we expect our theories to give us only approximately accurate models. Altering the notion of acceptance to take this into account, we end up with the notion advocated by Frisch. 
How does this sound in my idiolect? Well, each of Frisch's representational models of an inconsistent theory is just a model-theoretic model of one of the several theories that I see covering the same class of phenomena. So I don't need to bother about representational models. Of course, I would be well-advised to follow Frisch in requiring that in accepting a non-fundamental theory, one is only committed to the approximate accuracy of the theory's predictions. But this is of course the case with any less-thanfundamental theory, whether or not it is part of a team of theories that Frisch would see as forming an inconsistent theory. ${ }^{18}$

So I accept half of what Frisch argues for here: in delimiting the possible attitudes we might take towards non-fundamental theories I don't think we need to rely on the notion of representational models but I do think we should take partial accuracy of representation as our goal.

Here is a scorecard. I disagree with Frisch's claim that there exist inconsistent theories-but this is a merely terminological disagreement. I demur at seeing the phenomenon Frisch is interested in as providing an easy victory for the models-based account, and I don't believe that we need the distinction between representational and model-theoretic models. I do agree with him that in non-fundamental contexts we sometimes would rather have an inconsistent package than a consistent one, and that our epistemic attitudes turn on questions of approximate fit rather than perfect fit-but I think these last points have nothing special to do with the question of inconsistency and should

\footnotetext{
${ }^{18}$ Actually, I wonder whether van Fraassen would say that we can accept non-fundamental theories, given what he says about approximate truth (1980, p. 9). And I wonder whether I have much grasp of the notion of acceptance outside of van Fraassen's system. But this doesn't really matter: clearly we need to articulate a range of epistemic attitudes that one can take to non-fundamental theories, and clearly these are going to turn on approximate accuracy of models rather than perfect accuracy.
} 
occur to anyone who thinks about what we do with the non-fundamental theories that we keep on the books. And, most importantly, I am unconvinced by Frisch's argument that classical electrodynamics is an inconsistent theory.

\section{REFERENCES}

Bauer, G. and Dürr, D. 2001. “The Maxwell-Lorentz System of a Rigid Charge.” Annales Henri Poincaré 2 (2001): 179-196.

Feynman, R., Leighton, R. and Sands, M. 1964. The Feynman Lectures on Physics, Volume II. Reading, MA: Addison-Wesley.

Frisch, M. 2005. Inconsistency, Asymmetry, and Non-Locality: A Philosophical Investigation of Classical Electromagnetism. Oxford: Oxford University Press.

Hörmann, G. and Kunzinger, M. 2000. "Regularized Derivatives in a 2-Dimensional Model of Self-Interacting Fields with Singular Data." Zeitschrift für Analysis und ihre Anwendungen 19 (2000): 147-158.

Komech, A. and Spohn, H. 2000. "Long-Time Asymptotics for the Coupled MaxwellLorentz Equations." Communications in Partial Differential Equations 25 (2000): $559-584$.

Jackson, J. 1962. Classical Electrodynamics. New York: Wiley.

Spohn, H. 2004. Dynamics of Charged Particles and Their Radiation Field. Oxford: Oxford University Press.

Van Fraassen, B. 1980. The Scientific Image. Oxford: Oxford University Press.

Whittaker, E. 1947. A Treatise on the Analytical Dynamics on Particles and Rigid Bodies, $4^{\text {th }}$ edition. Cambridge: Cambridge University Press. 